\title{
COGENERACIÓN SOLAR UTILIZADA PARA PROCESOS EN LA INDUSTRIA ALIMENTARIA*
}

\author{
Christian André De Montreuil Estupiñan \\ Universidad Nacional Mayor de San Marcos \\ Erick Eduardo Vargas Guevara \\ Pontificia Universidad Católica del Perú
}

\begin{abstract}
Resumen: Mediante la investigación que da origen a este artículo se busca demostrar la viabilidad técnica, financiera y legal del uso de la cogeneración solar para el sector de industrias alimentarias en nuestro país; para esto se procederá con una cuantificación de la energía eléctrica y térmica obtenida mediante un ciclo Rankine convencional, con la particularidad de que el agua usada para condensar el vapor saliente de la turbina será utilizada para los procesos requeridos en las plantas industriales, debido a la elevada temperatura obtenida del intercambio térmico con el vapor. Todo ello se realizará detallando los procesos en los que se aprovechará la energía obtenida y la cantidad de equipos a utilizar en este sistema, así como las especificaciones necesarias y parámetros termodinámicos para el correcto funcionamiento del sistema de cogeneración. Además, demostraremos la viabilidad legal de optar por un sistema de cogeneración solar en el ordenamiento jurídico peruano, y, en vista de la importancia de este proyecto, plantearemos un esquema legal y administrativo por medio del cual puede ser llevado a cabo. En ese sentido, el proyecto es interdisciplinario, pues implica la conjunción de planteamientos jurídicos y de ingeniería.
\end{abstract}

Palabras Clave: Cogeneración solar. Energías renovables. Energía solar. Termosolar. Energía térmica. Procesos industriales. Promoción de inversiones. 


\title{
Solar cogeneration for processes in the food industry
}

\begin{abstract}
In this paper, the technical, financial and legal feasibility of employing solar cogeneration for the food industry sector in Peru will be demonstrated. In consequence, we will quantify the electric and thermal energy obtained from a conventional Rankine cycle, with the particularity that the water used to condense the steam that exits the turbine will be employed for the required processes in the industrial plants, because of the high temperature obtained from the heat exchange with the steam. In addition, we will detail the processes in which the obtained energy will be employed, the quantity of devices to be used in this system, as well as the required specifications and the thermodynamic parameters, for a proper operation of the cogeneration system. Moreover, we will prove the legal feasibility of choosing a solar cogeneration system in the Peruvian legal system, and, in view of the relevance of this project, we will propose an administrative and legal scheme through which it can be accomplished. In this respect, the project is interdisciplinary, because it involves the combination of legal and engineering approaches.
\end{abstract}

Keywords: Solar Cogeneration. Renewable Energies. Solar Energy. Thermosolar. Thermal Energy. Industrial Processes. Investment Promotion.

Artículo basado en la ponencia presentada en el II Congreso Latinoamericano de Investigación y Educación Superior Interdisciplinaria (IEI 2018), Mesa temática 3 Educación superior interdisciplinaria: metodologías, resultados, desafíos, realizado en Lima del 18 al 21 de septiembre de 2018.

Queremos agradecer al ingeniero César Alejandro Quispe Gonzáles, Doctor en Ingeniería Mecánica y docente de la Escuela Académica Profesional de Ingeniería Mecánica de Fluidos de la Universidad Nacional Mayor de San Marcos (UNMSM), por la asesoría y apoyo brindados en la elaboración de la parte técnica del presente artículo. 


\section{Christian André De Montreuil Estupiñan}

Ingeniero Mecánico de Fluidos por la Universidad Nacional Mayor de San Marcos (UNMSM), con especialidad en Termofluidos. Miembro del Grupo de Investigación "MECFLUET" de la UNMSM en las líneas de investigación de energética, termofluidos y sistemas de control de emisiones. Ingeniero de Proyectos en la Dirección General de Programas y Proyectos de Transportes en el Ministerio de Transportes y Comunicaciones. Asistente de docencia en la Escuela Académico Profesional de Ingeniería Mecánica de Fluidos de la UNMSM.

\section{Correo electrónico: ademontreuile@gmail.com}

\section{Erick Eduardo Vargas Guevara}

Abogado por la Pontificia Universidad Católica del Perú (PUCP). Jefe de práctica de Introducción a las Ciencias Jurídicas en la Facultad de Derecho de la PUCP. Ha sido adjunto de docencia de Derecho Administrativo II y Derecho de las Telecomunicaciones en la misma casa de estudios. Primer puesto del XXII Curso de Extensión Universitaria en Regulación de OSIPTEL. Asociado de Quiñones Alayza Abogados.

Correo electrónico: evargasg@pucp.pe 


\section{Introducción}

Frente a los actuales niveles de contaminación mundial, la obtención de energía mediante recursos renovables tiene cada vez mayor prioridad, siendo la fuente solar una de las de más potencial. Otra solución es la tecnología de la cogeneración, cuya principal virtud es el aprovechamiento de la energía térmica residual de la obtención de energía eléctrica.

Por eso, en la presente investigación caracterizamos el sistema energético de la cogeneración solar como una alternativa de producción de energía limpia, ambientalmente amigable, renovable e inagotable, y demostramos su viabilidad técnica, financiera y legal. Elegimos para este fin la industria láctea, por ser básica para el desarrollo humano.

\section{Una nueva forma de aprovechamiento energético como solución a un problema global}

El cambio climático es un problema global que se manifiesta en el ascenso de las temperaturas, el incremento de la frecuencia de ciertos fenómenos climáticos extremos, el retroceso de los glaciares y consiguiente subida del nivel del mar, y el aumento de concentraciones en la atmósfera de gases de efecto invernadero (GEI). Resulta entonces necesario explorar nuevas formas de producción y utilización de la energía (González 2011).

\subsection{El rol de los recursos energéticos renovables frente al cambio climático}

Según el Objetivo 7 de los Objetivos de Desarrollo Sostenible de las Naciones Unidas - Garantizar el acceso a una energía asequible, segura, sostenible y moderna para todos-para proteger el medio ambiente es necesario invertir en fuentes de energía limpia como la solar (PNUD s.a.). Esta exigencia legitima la actuación de las administraciones públicas (Mora 2014). Así, los esfuerzos contra el cambio climático pasan, necesariamente por la potenciación de las energías renovables (Alenza et al. 2017). ${ }^{1}$ 
Entre las ventajas de los recursos energéticos renovables (RER), tenemos: (1) una casi nula emisión de $\mathrm{CO}_{2}$ y otros $\mathrm{GEI}$ por cada MWh generado, en comparación con la energía fósil; (2) la factibilidad técnica que tienen para generar energía eléctrica en zonas aisladas y vulnerables, favoreciendo el acceso a la energía; y (3) el permitir la diversificación de la matriz eléctrica y disminuir la dependencia del suministro eléctrico (OSINERGMIN 2016).

Tratados internacionales como el Protocolo de Kyoto de la Convención Marco de las Naciones Unidas sobre el Cambio Climático de 1988, el Acuerdo de Copenhague de 2009, los acuerdos de la Plataforma de Durban de 2012, el Acuerdo de París de 2015 y la Proclamación de Marrakech de 2016, enfrentan el problema del cambio climático, enfatizando en el uso de los RER para tal fin.

En el artículo 22, inciso 2, de la «Constitución Política del Perú» de 1993, se reconoce el derecho de toda persona «a la paz, a la tranquilidad, al disfrute del tiempo libre y al descanso, así como a gozar de un ambiente equilibrado y adecuado al desarrollo de su vida». Sobre esta base, el Estado peruano ha buscado promover la inversión en la generación eléctrica con RER con la emisión del Decreto Legislativo $1002^{2}$ (2008) y de su Reglamento, aprobado por Decreto Supremo 12-2011-EM, normas donde se contempla la energía solar como un tipo de RER.

La energía solar es una de las fuentes más limpias de energía, pues no produce emisiones de efecto invernadero (Solangi et al. 2011). ${ }^{3}$ Este tipo de energía es obtenida mediante la captación de la luz y del calor emitidos por el sol, existiendo dos tipos: fotovoltaica y termoeléctrica (Dammert et al. 2017). Un tipo de energía solar termoeléctrica, la tecnología termosolar de concentración, es usada «[...] para producir electricidad con un ciclo termodinámico convencional a partir de un fluido calentado a alta temperatura» (OSINERGMIN 2017: 35).

Debido al importante rol de los RER en la mitigación de los efectos del cambio climático, en la reciente Ley 30754 (2018), «Ley Marco sobre Cambio Climático», artículo 16, se ordenó un rol activo del Estado en implementar programas, proyectos y actividades orientadas al cambio progresivo de los modelos de consumo y de la matriz energética a energías renovables y limpias.

2 Decreto Legislativo de promoción de la inversión para la generación de electricidad con el uso de energías renovables.

3 Por ejemplo, la primera planta termosolar en construcción en Latinoamérica, el complejo solar Cerro Dominador (210 MW), que combinará 100 MW de energía fotovoltaica y $110 \mathrm{MW}$ de energía de concentración solar, evitará la emisión de unas 870,000 toneladas de $\mathrm{CO}_{2}$ por año (RevistaEnergía.pe 2018). 


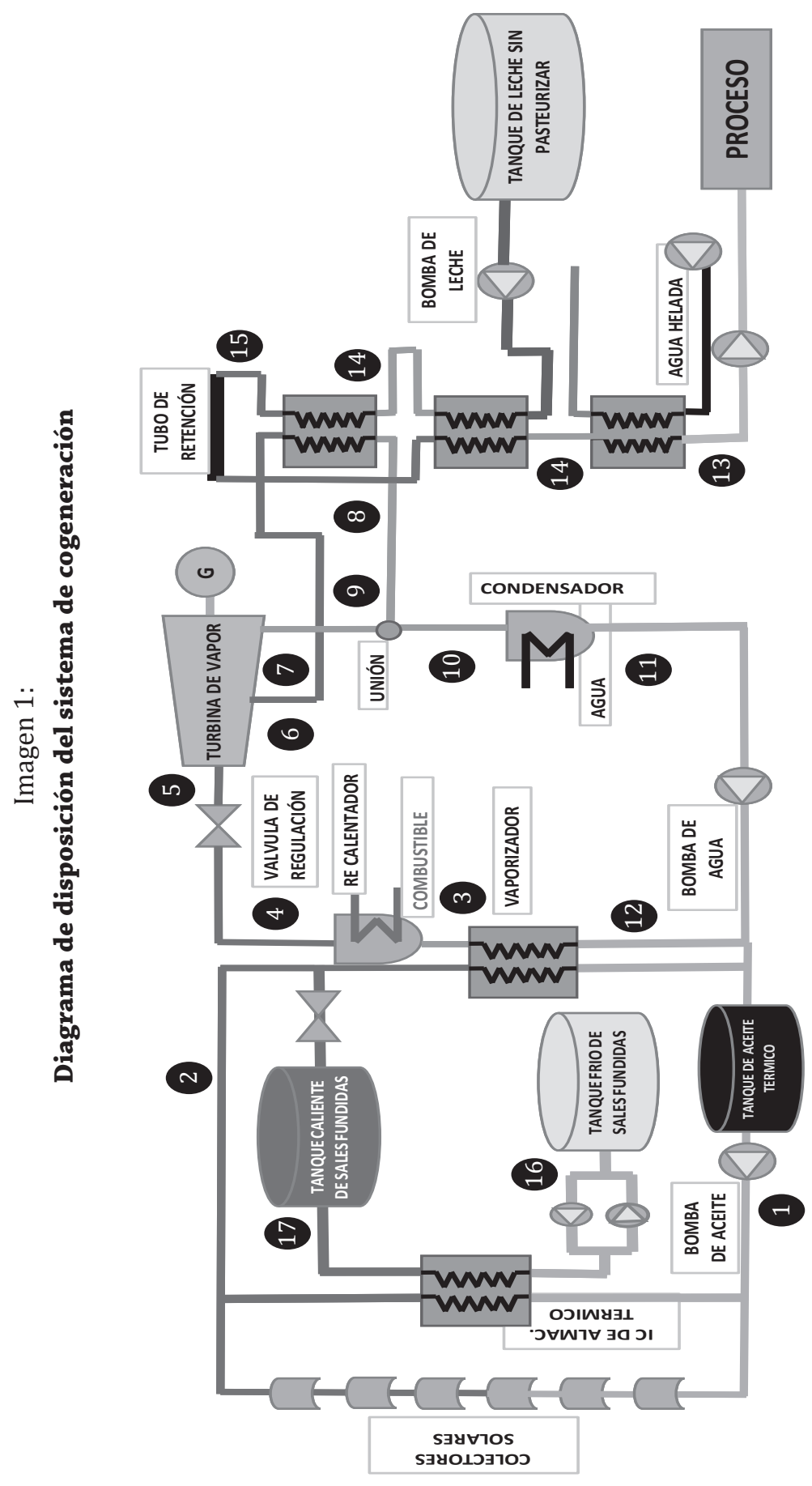

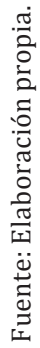




\subsection{La tecnología de la cogeneración como solución}

Los sistemas de cogeneración tienen una eficiencia energética mayor en comparación con las técnicas convencionales de producción de electricidad. Esto se explica, según señala E. López debido a que requieren para producir la misma cantidad de energía útil, una cantidad de energía primaria menor que una instalación convencional (2000). ${ }^{4}$

En el Perú, la Ley 28832 (2006), «Ley para Asegurar el Desarrollo Eficiente de la Generación Eléctrica», reconoce expresamente en el artículo 1, numeral 5, a la cogeneración como una forma de generación de energía eléctrica y la define como un «Proceso de producción combinada de energía eléctrica y energía térmica, ${ }^{5}$ que hace parte integrante de una actividad productiva, en el cual la energía eléctrica está destinada al consumo propio o de terceros». Además, esta tecnología cuenta con su propia reglamentación, expedida mediante el Decreto Supremo 037-2006-EM.

\section{La cogeneración solar utilizada para procesos e mitigación del cambio climático, a partir de la industria de leche en el Perú}

Nuestro proyecto, que cuenta con elementos de cogeneración y de energía solar, busca mitigar los GEI mediante un campo de concentradores solares parabólicos ${ }^{6}$ que calientan un fluido secundario que será de ayuda en la vaporización del fluido de trabajo para la generación de energía eléctrica, y, a su vez, en la pasteurización de la leche. Este último es un proceso fundamental en la industria láctea ya que erradica los microorganismos y bacterias que pueden hacer nocivo el producto.

La generación de energía eléctrica se realizará mediante un ciclo Rankine equipado por dos intercambiadores de calor para calentar el agua, que serán: el que use la energía térmica de los captadores solares y una caldera secundaria que socorra al sistema; así se podrá reducir la cantidad

4 Por ejemplo, en España se fomenta la cogeneración mediante Real Decreto 616/2007, para reducir la demanda de energía mediante medidas económicamente viables o favorables para el medio ambiente (art. 8). En dicho país la cogeneración tiene una fuerte presencia en la industria agrícola, alimentaria y del tabaco, que concentraron el 19.4\% de la potencia instalada en el año 2013 (Ministerio de Industria, Energía y Turismo 2016).

$5 \quad$ El calor útil.

6 Para lo cual se tomaron ideas del Observatorio de Energía Renovable para América Latina y Caribe (2013). 
de captadores necesarios y, por ende, la inversión inicial que esto conlleva. Cabe resaltar que este criterio dependerá de la empresa que esté interesada en concretar esta iniciativa.

\subsection{Descripción del sistema}

El ciclo de generación de energía que describimos a continuación comprende las siguientes partes:

\section{- Campo de concentración solar}

Está compuesto principalmente por los captadores solares de tipo cilindros parabólicos, que elegimos por su amplio uso. Se hace pasar un flujo de aceite a través de estos, lo que conllevará a su elevación de temperatura para posteriormente ser dividido en dos flujos de diferente proporción, siendo el de mayor proporción el encargado de ceder calor a un flujo de agua que se utilizará como fluido de trabajo para la generación de energía eléctrica en nuestra planta de $18 \mathrm{MW}$. El otro flujo de aceite será destinado al almacenamiento térmico. Una vez cedido el calor en ambos flujos, el aceite vuelve a comenzar el ciclo de calentamiento en los captadores solares; además de ello, este sistema incluye el tanque de aceite donde suelen almacenarse las bombas que llevan el aceite a una presión que venza todas las pérdidas de fricción y locales que se encuentren en el recorrido de tuberías e intercambiadores de calor.

\section{- Cuarto de máquinas}

Está compuesto por los componentes de un ciclo Rankine convencional. La caldera solo se usa de manera complementaria, ya que, al diseñar un sistema de generación de tan solo $18 \mathrm{MW}$ no se considera que funcione por completo con energía termosolar, pues demandaría una mayor inversión inicial que sería sustentada si la potencia fuera mucho mayor. La turbina tiene un comportamiento tanto de contrapresión como de condensación, porque se extrae un flujo de media presión (300 kPa) para su aprovechamiento para la pasteurización. El resto del flujo se extrae a una presión por debajo de la presión atmosférica $(50 \mathrm{kPa})$.

\section{- Almacenamiento térmico}

Se considera que el almacenamiento térmico se dé con sales de nitrito, ya que pueden llegar a altas temperaturas como $500^{\circ} \mathrm{C}$ sin sufrir un cambio de fase, 
lo cual ayuda a un óptimo almacenamiento térmico. Se puede apreciar que este sistema consta de un intercambiador de calor que pone en contacto el flujo de aceite y de sales de nitrito. Además, se diseña el tanque de sales con el volumen necesario para cubrir los tiempos de mayor radiación.

\section{- $\quad$ Pasteurización de leche}

Se consideran 10 pasteurizadores de 5,000 L/hr de capacidad, siendo utilizado el vapor saliente a $300 \mathrm{kPa}$ para su calentamiento de leche tibia $\left(38^{\circ} \mathrm{C}\right)$ a leche caliente $\left(72^{\circ} \mathrm{C}\right)$. Se tiene también como objetivo dejar la leche a esta temperatura por 15 segundos para la eliminación de todas las bacterias a través del tubo de retención. Una vez purificada, la leche es enviada otro intercambiador de calor que aprovecha el flujo de leche a temperatura fría, para volver el flujo purificado a una temperatura tibia $\left(36^{\circ} \mathrm{C}\right.$ ) (Domínguez et al. 2013). Posteriormente, se requiere llevarla a una temperatura de $4^{\circ} \mathrm{C}$, por lo que es necesaria la implementación de un sistema de refrigeración; en este caso, por chiller, el cual tiene que cumplir con la cantidad de calor necesaria para lograr el enfriamiento debido.

Además de ello, la numeración en la figura servirá de referencia para los cálculos e identificación en nuestra tabla de esquema termodinámico posteriormente mostrado en nuestro trabajo.

\subsection{Principales parámetros a considerar}

En la formulación de este proyecto, se han llegado a identificar como parámetros de diseño más importantes:

\subsubsection{Irradiancia solar}

El cálculo de este parámetro sirve para analizar el potencial que tiene cada región para la implementación de estos sistemas de captación solar, siendo de importancia ya que permite determinar dónde y cuándo un sistema solar térmico o fotovoltaico tiene mejor aprovechamiento. Los aspectos a tomar en cuenta son:

- Ángulo cenital.

- Cantidad de horas de radiación.

- $\quad$ Ángulo horario.

- $\quad$ Orientación y tipo de seguimiento.

- $\quad$ Latitud y longitud del lugar. 


\subsubsection{Tipo de captación solar}

Debido a su gran flexibilidad, el sistema de captación será de tipo cilíndrico parabólico. Esta cualidad hace que su uso sea mayoritario en las plantas solares térmicas. Este sistema tiene las siguientes variables de diseño:

- $\quad$ Ancho y largo del colector.

- Distancia focal.

- $\quad$ Diámetro mínimo del tubo receptor.

- Pérdidas geométricas.

- $\quad$ Reflectividad de reflector.

- $\quad$ Transmisividad y absortividad del absorbedor.

- Reflectancia espectral y factor de interpretación (Alegría 2016; Paredes 2012).

\subsubsection{Potencia eléctrica generada}

Se está considerando una planta de $15 \mathrm{MW}$ de potencia útil, valor resultante al restar la energía demandada por la bomba en el ciclo Rankine de la energía obtenida por el trabajo de la turbina. Se considera:

- $\quad$ Tipo de turbina utilizada.

- $\quad$ Presiones de entrada y salida de la turbina.

- $\quad$ Eficiencia adiabática, mecánica y del generador.

\subsubsection{Energía térmica para pasteurización}

Al ser este un proyecto de cogeneración, se busca un proceso para el aprovechamiento de la energía, siendo en este caso la pasteurización, con las siguientes variables:

- Cantidad de leche procesada.

- $\quad$ Temperatura de leche fría y caliente.

- $\quad$ Presión de entrada del vapor.

- Temperatura a enfriar.

- $\quad$ Potencia del chiller necesaria para el enfriamiento.

\subsection{Metodología de cálculo}

Se parte con los cálculos de radiación solar, tomando como referencia la radiación del sol a la tierra, siendo esta constante de $G=1366 \mathrm{~W} / \mathrm{m}^{2}$ 
Luego se toma la ciudad de Arequipa como referencia. De esta manera, se tendrían las siguientes consideraciones:

Latitud $\lambda=-16^{\circ} 23^{\prime} 56^{\prime \prime}$ ó $-16.40^{\circ}$

Para simplificación del cálculo, se convierte el valor a radianes

$$
\lambda=-16.40^{\circ} \times\left(\pi \mathrm{rad} / 180^{\circ}\right)=-0.2862 \mathrm{rad}
$$

Declinación Media $\delta=23.45$

$$
\delta=23.45 \times \operatorname{seno}\left(\frac{360 \times(284+n)}{365}\right)
$$

Siendo $n$ el número de día del año, pudiendo representar el promedio de cada mes con los siguientes valores:

$\mathrm{n}=17,47,75,105,135,162,198,228,258,288,318,344$

Realizando el cálculo

$$
\delta=-20.92^{\circ}=-0.365 \mathrm{rad}
$$

Ángulo horario $\tau$

$$
\tau=15^{\circ} \times(h-12)
$$

Siendo $h$ la hora del día, se toma como referencia que las horas efectivas de radiación son desde las 7 hasta las 17, ya que el ángulo $\tau$ debe estar entre $-90^{\circ}$ y $90^{\circ}$.

Para el cálculo del ángulo $\theta$, la opción que usaremos consiste en que el colector gire sobre el eje horizontal Norte-Sur con ajuste continuo, siendo la siguiente fórmula:

$$
\cos \left(\theta_{S}\right)=\sqrt{(\sin \lambda \times \sin \delta+\cos \lambda \times \cos \delta \times \cos \tau)^{2}+\cos ^{2} \delta \times \operatorname{sen}^{2} \tau}
$$
fórmula:

Para el cálculo de la radiación directa $I_{0}$, se considera la siguiente

$$
I_{0(\text { media })}=1230 \times \mathrm{e}^{\wedge}\left(\frac{-1}{3.8 \times \cos \left(\theta_{S}-1.6^{\circ}\right)}\right)
$$


Teniendo la radiación media, se tiene que considerar el valor del rendimiento óptimo del captador, el cual está en base a los materiales de este.

$$
\begin{gathered}
\eta_{\text {optica }}=\rho \times \tau \times \alpha \times \mathrm{d} \times \gamma \\
\eta_{\text {optico }}=0.9 \times 1 \times 0-95 \times 0.87 \times 0.96=0.714
\end{gathered}
$$

Se considera que el diseño se plantee en el primer mes el año, enero, pudiendo mostrar los valores calculados en el día 17 del año.

Tabla 1:

\section{Radiación media en enero}

\begin{tabular}{|c|c|c|c|c|c|}
\hline Hora & Ángulo horario $\tau$ & Declinación $\boldsymbol{\delta}$ & Latitud $\boldsymbol{\lambda}$ & $\cos \boldsymbol{\theta}$ & Io $\left(\mathbf{W} / \mathbf{m}^{2} \mathbf{~}\right.$ \\
\hline 7 & -1.31 & -0.365 & -0.2862 & 0.9617 & 937.46 \\
\hline 8 & -1.05 & -0.365 & -0.2862 & 0.9776 & 941.13 \\
\hline 9 & -0.79 & -0.365 & -0.2862 & 0.9878 & 943.33 \\
\hline 10 & -0.52 & -0.365 & -0.2862 & 0.9935 & 944.47 \\
\hline 11 & -0.26 & -0.365 & -0.2862 & 0.9961 & 944.96 \\
\hline 12 & 0.00 & -0.365 & -0.2862 & 0.9969 & 945.08 \\
\hline 13 & 0.26 & -0.365 & -0.2862 & 0.9961 & 944.96 \\
\hline 14 & 0.52 & -0.365 & -0.2862 & 0.9935 & 944.47 \\
\hline 15 & 0.79 & -0.365 & -0.2862 & 0.9878 & 943.33 \\
\hline 16 & 1.05 & -0.365 & -0.2862 & 0.9776 & 941.13 \\
\hline 17 & 1.31 & -0.365 & -0.2862 & 0.9617 & 937.46 \\
\hline & & PROMEDI0 & & 942.53 \\
\hline
\end{tabular}

Fuente: Elaboración propia

Realizando el mismo procedimiento para todos los meses, se puede apreciar la tendencia de la irradiancia promedio en la siguiente gráfica: 
Gráfica 1:

Arequipa: nivel de irradiancia $(\mathrm{kW})$ por mes en un año común

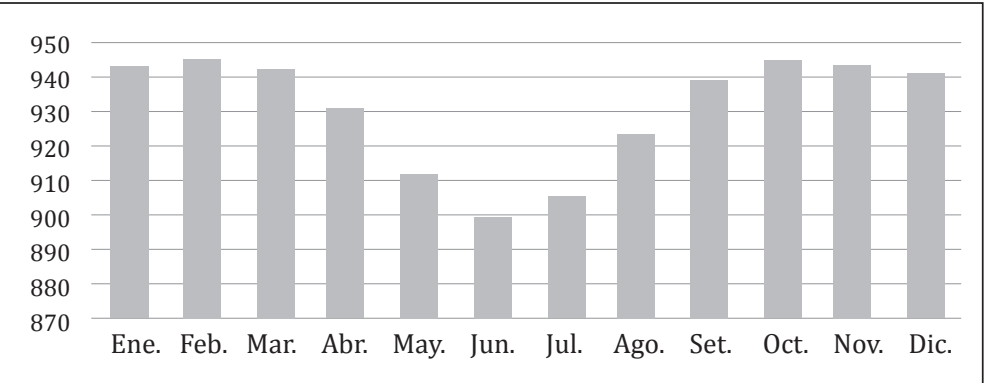

Fuente: Elaboración propia

Una vez obtenida la irradiancia, se diseña o selecciona un panel parabólico. Tomaremos como referencia el Panel Eurotrough 150, ya que cumple con los mejores estándares de diseño y se encuentra posicionado en el mercado. Además, seleccionaremos como aceite térmico el Therminol 62 (Therminol 2015).

El rendimiento óptico en el Panel Eurotrough 150 es el siguiente (Lüpfert et al. 2003):

$$
\begin{gathered}
\eta_{\text {optica }}=\rho \times \tau \times \alpha \times \gamma \\
\eta_{\text {optico }}=0.93 \times 0.95 \times 0.95 \times 0.91=0.764
\end{gathered}
$$

Siendo:

$\rho:$ Reflectividad de los espejos.

$\tau$ : Transmisividad del vidrio absorbedor.

$\alpha$ : Absortancia del recubrimiento selectivo.

$\gamma$ : Factor de interceptación.

Las funciones de la densidad y el calor específico en relación a la temperatura del aceite térmico son (Therminol 2015):

$$
\rho(\mathrm{T})=973.01-0.7879 \times \mathrm{T}^{\mathrm{kg}} / \mathrm{m}^{3} \quad \mathrm{Cp}(\mathrm{t})=1.9137-0.0021 \times \mathrm{T} \mathrm{kJ} / \mathrm{kg} . \mathrm{K}
$$

Considerando $\mathrm{T}$ como el valor medio entre los valores máximo y mínimo de las temperaturas en el aceite a la entrada y salida del campo solar, y siendo: 


$$
\begin{gathered}
T_{\text {min }}=190^{\circ} \mathrm{C} \quad T_{\text {máx }}=315^{\circ} \mathrm{C} \\
T_{\text {medio }}=252.5^{\circ} \mathrm{C}
\end{gathered}
$$

En relación a la velocidad del aceite, es importante considerar que el flujo debe ser — de preferencia- de régimen turbulento, ya que se requiere que el tubo absorbedor que contendrá dicho flujo sea refrigerado en su interior, por lo que se debe considerar un valor del número de Reynolds mínimo de $2 \times 10^{5}$. Cabe resaltar que la potencia solar decrece en meses de invierno, por lo que también lo haría el número de Reynolds, así que, con el fin de asegurar un valor mínimo de $2 \times 10^{5}$ durante todo el año, se toma un valor mucho mayor a este en caso se diseñe considerando un mes de verano. Dado que el presente trabajo toma como referencia el mes de enero, se fijará un valor en enero de $4 \times 10^{6}$, por lo que, planteando y reemplazando la fórmula, se podrá hallar el flujo másico:

$$
R e=\frac{v \times \rho \times D}{\mu}
$$

Cabe resaltar que el diámetro de la tubería y la viscosidad del aceite se encuentra en la ficha técnica del Eurotrough 150 (Lüpfert et al. 2003) y Therminol 62 (Therminol 2015) respectivamente.

$$
\begin{gathered}
4 \times 10^{6}=\frac{v \times 774.065 \mathrm{~kg} / \mathrm{m}^{3} \times 0.065 \mathrm{~m}}{0.000325 \frac{\mathrm{kg}}{\mathrm{m} . \mathrm{s}}} \\
v=2.58 \mathrm{~m} / \mathrm{s}
\end{gathered}
$$

Teniendo la velocidad y el diámetro de la tubería, se podrá hallar el flujo másico:

$$
\begin{gathered}
\dot{m}=v \dot{\times} D \times \rho \\
\dot{m}=2.58 \mathrm{~m} / \mathrm{s} \times 0.065 \mathrm{~m} \times 774.065 \mathrm{~kg} / \mathrm{m}^{3}=6.637 \mathrm{~kg} / \mathrm{s}
\end{gathered}
$$

Se realiza el cálculo de las pérdidas térmicas:

Pérdidas $Q \rightarrow a m b$

$$
\begin{aligned}
& =0.0019 \times\left(T_{\text {medio }}-T_{a m b}\right)^{2}+0.405 \times\left(T_{\text {medio }}-T_{\text {amb }}\right)+24.9 \\
& \times\left[\left(\frac{I_{o}}{900}\right) \times \cos (\theta)-1\right]
\end{aligned}
$$


Considerando $T_{a m b}=25^{\circ} \mathrm{C}$ y, reemplazando, se obtiene:

$$
\text { Perdidas }_{Q \rightarrow a m b}=28.19 \mathrm{~kW}
$$

Pudiendo, finalmente, obtener la potencia generada por panel:

Potencia $_{\text {panel }}=$ Area $_{\text {panel }} \times I_{o} \times \cos (\theta) \times \eta_{\text {optico }} \times \eta_{\text {geómetrico }} \times F_{e}-$ Perdidas $_{Q \rightarrow a m b}$

Cabe resaltar que se tiene la eficiencia geométrica y el área del panel de los datos del fabricante y que se está considerando el Factor de ensuciamiento $F_{e}=0.95$. Entonces: Potencia ${ }_{\text {panel }}=496.21 \mathrm{~kW}$

$$
\text { Potencia }_{\text {panel }}=496.21 \mathrm{~kW}
$$

Teniendo este dato, podemos hallar la cantidad de paneles solares necesarios en una fila para poder cubrir la diferencia de temperatura entre el lado frío y caliente del sistema de captación solar; utilizando la fórmula para calcular la temperatura elevada en el recorrido de un panel.

$$
\begin{gathered}
\text { Potencia }_{\text {panel }}=\dot{m} \times \int_{252.5}^{T} C p d T \\
496.21 \mathrm{~kW}=6.637 \mathrm{~kg} / \mathrm{s} \times \int_{252.5}^{T} 1.9137+0.0021 \times \mathrm{T} d T
\end{gathered}
$$

Siendo:

$$
T=282.71^{\circ} \mathrm{C}
$$

Por lo que la diferencia de temperatura sería la siguiente:

$$
\Delta T=282.71-252.5=30.21^{\circ} \mathrm{C}
$$

Entonces, se puede hallar el número de paneles por fila:

$$
N_{\text {Paneles }}=\frac{315^{\circ} \mathrm{C}-190^{\circ} \mathrm{C}}{30.21^{\circ} \mathrm{C}}=4.14 \cong 4 \text { paneles }
$$

Para hallar el número de filas a usar, se tiene que hacer un análisis con la potencia requerida en la planta de generación eléctrica. Se está considerando una potencia útil de la planta de $18 \mathrm{MW}$, con las siguientes consideraciones en sus parámetros: 


\section{Parámetros de la planta de generación eléctrica}

\begin{tabular}{|l|c|}
\hline Eficiencia adiabática de la turbina & $85 \%$ \\
\hline $\begin{array}{l}\text { Eficiencia adiabática de la bomba de } \\
\text { alimentación: }\end{array}$ & $85 \%$ \\
\hline $\begin{array}{l}\text { Eficiencia mecánica (eje de transmisión turbina } \\
\text { generador): }\end{array}$ & $90 \%$ \\
\hline Eficiencia del generador: & $95 \%$ \\
\hline Caída de presión en intercambiador de calor & $68.95 \mathrm{kPa}=10 \mathrm{psi}$ \\
\hline Caída de presión en el condensador: & $10 \% \mathrm{de}$ entrada \\
\hline Poder calorífico del gas natural: & 0.3 \\
\hline $\begin{array}{l}\text { La energía de los captadores solares vaporizará } \\
\text { el agua hasta llevarla a un estado de vapor } \\
\text { saturado con una calidad de }\end{array}$ & $9,200 \mathrm{Kcal} / \mathrm{m}^{3}=38,493 \mathrm{~kJ} / \mathrm{kg}$ \\
\hline $\begin{array}{l}\text { Una caldera cumple la función de recalentador } \\
\text { para llevar el vapor húmedo a estado de vapor } \\
\text { sobrecalentado. }\end{array}$ & \\
\hline
\end{tabular}

Parámetros de la planta de pasteurización

\begin{tabular}{|l|c|}
\hline Temperatura fría de la leche: & $4^{\circ} \mathrm{C}$ \\
\hline Temperatura caliente de la leche: & $72^{\circ} \mathrm{C}$ \\
\hline Cantidad de leche por pasteurizador: & $500 \mathrm{~L} / \mathrm{h}$ \\
\hline Cantidad de pasteurizadores: & 10 \\
\hline Calor especifico de la leche: & $3.8937 \mathrm{~kJ} / \mathrm{kg} \cdot{ }^{\circ} \mathrm{C}$ \\
\hline Densidad de la leche: & $1.032 \mathrm{~kg} /$ \\
\hline
\end{tabular}


Teniendo en cuenta los parámetros termodinámicos que están en la Tabla 2 (ver página 156-157), podemos hallar las potencias generadas de la turbina y la bomba en el ciclo de generación eléctrica.

- $\quad$ Potencia generada en la turbina por el flujo de vapor saliente a 300 kPa hacia el pasteurizador antes de que sea extraída.

$$
\begin{gathered}
W_{T 1}=\left(h_{5}-h_{6}\right) \times \dot{m}_{6} \times \eta_{\text {mecánica }} \times \eta_{\text {generador }} \\
W_{T 1}=(2796 \mathrm{~kJ} / \mathrm{kg}-2564.97 \mathrm{~kJ} / \mathrm{kg}) \times 0.87 \mathrm{~kg} / \mathrm{s} \times 0.90 \times 0.95 \\
W_{T 1}=171.32 \mathrm{~kW}
\end{gathered}
$$

- $\quad$ Potencia generada en la turbina por el flujo de vapor que se extrajo a $50 \mathrm{kPa}$ que se llevó posteriormente al condensador.

$$
\begin{gathered}
W_{T 2}=\left(h_{5}-h_{7}\right) \times \dot{m}_{7} \times \eta_{\text {mecánica }} \times \eta_{\text {generador }} \\
W_{T 2}=(2796 \mathrm{~kJ} / \mathrm{kg}-2337.94 \mathrm{~kJ} / \mathrm{kg}) \times 45.74 \mathrm{~kg} / \mathrm{s} \times 0.90 \times 0.95 \\
W_{T 2}=17,912.39 \mathrm{~kW}
\end{gathered}
$$

- Potencia demandada en la bomba por elevar la presión del flujo de agua desde 45 a $1568.95 \mathrm{kPa}$ a su posterior llegada a la caldera

$$
\begin{gathered}
W_{B}=v_{11} \times\left(P_{12}-P_{11}\right) \times \dot{m}_{5} \\
W_{B}=0.0010018 \mathrm{~m}^{3} / \mathrm{kg} \times(1568.95 \mathrm{kPa}-45 \mathrm{kPa}) \times 46.60 \mathrm{~kg} / \mathrm{s} \\
W_{B}=83.71 \mathrm{~kW}
\end{gathered}
$$

Por lo que:

$$
\begin{gathered}
W_{U t i l}=171.32 \mathrm{~kW}+17,912.39 \mathrm{~kW}-83.71 \mathrm{~kW} \\
W_{U t i l}=18,000 \mathrm{~kW}
\end{gathered}
$$

Pudiendo demostrar que la potencia útil es igual a 18 MW.

Para seguir con el cálculo de la cantidad de filas de captadores solares, se determina la potencia requerida en el vaporizador: 
REvista KAWSAYPACHA: SOCIEDAd y MEdio AMBIENTE Nº 3 (2019)

\begin{tabular}{|c|c|c|c|c|c|c|c|c|c|c|c|c|c|c|c|}
\hline \multirow{11}{*}{ 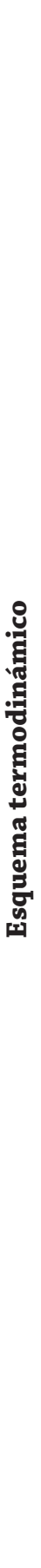 } & 冚 & 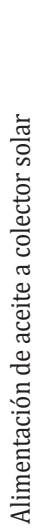 & 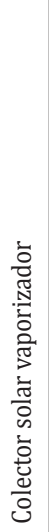 & 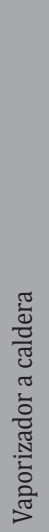 & 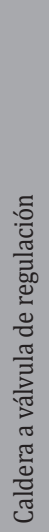 & 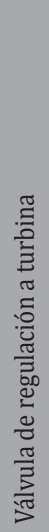 & 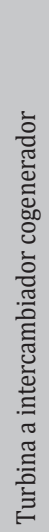 & 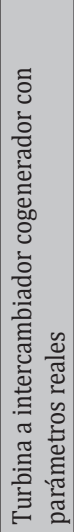 & 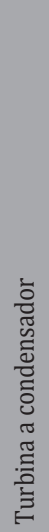 & 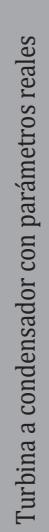 & 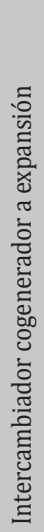 & 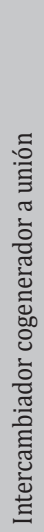 & 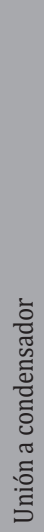 & 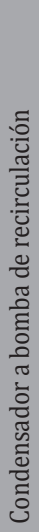 & 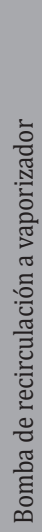 \\
\hline & $\Xi \frac{\tilde{n}}{20}$ & 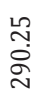 & 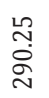 & $\begin{array}{l}0 \\
\dot{q}\end{array}$ & $\begin{array}{l}0 \\
\dot{b}\end{array}$ & $\begin{array}{l}0 \\
\dot{b}\end{array}$ & 用 & $\hat{\infty}$ & ஸे & 官 & $\hat{\infty}$ & $\hat{\infty}$ & $\begin{array}{l}b \\
\dot{q}\end{array}$ & $\begin{array}{l}0 \\
\text { ơ }\end{array}$ & $\begin{array}{l}0 \\
\dot{\sigma}\end{array}$ \\
\hline & $\stackrel{0}{0}$ & 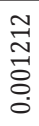 & 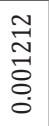 & & & & & & & & & & & & \\
\hline & $\underbrace{\stackrel{000}{3}}_{>}$ & & & 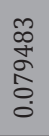 & $\begin{array}{l}\stackrel{+}{\sim} \\
\underset{\sim}{二} \\
\end{array}$ & 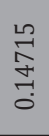 & 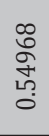 & 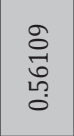 & 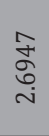 & $\begin{array}{l}\text { ח } \\
\infty \\
\infty \\
\infty \\
\text { i }\end{array}$ & 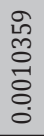 & 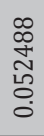 & $\begin{array}{l}\text { ô } \\
\text { ti } \\
\text { ì }\end{array}$ & 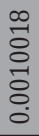 & $\begin{array}{l}\overrightarrow{-} \\
\overline{8} \\
8 \\
- \\
8 \\
0 \\
0\end{array}$ \\
\hline & $x$ & & & 草 & & , & $\begin{array}{l}\text { Ñ} \\
\text { ᄋ̊ } \\
\tilde{0}\end{array}$ & 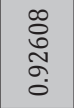 & $\begin{array}{l}\text { ज्ञ } \\
\text { חె } \\
\infty \\
0\end{array}$ & $\begin{array}{l}\infty \\
0 \\
ర \\
ٍ \\
\infty \\
0\end{array}$ & , & $\begin{array}{l}\text { O̊ } \\
\stackrel{0}{0}\end{array}$ & $\begin{array}{l}\stackrel{L}{\infty} \\
\infty \\
0\end{array}$ & , & , \\
\hline & $\underbrace{0}_{=}$ & $\stackrel{\circ}{\circ}$ & $\begin{array}{l}\overrightarrow{0} \\
\infty \\
0 \\
\stackrel{-1}{m}\end{array}$ & $\begin{array}{l}m \\
\infty \\
\infty\end{array}$ & 요 & 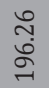 & $\begin{array}{l}\text { m } \\
\text { ñ } \\
m \\
n\end{array}$ & $\begin{array}{l}m \\
\text { ஸे } \\
\text { m} \\
\sim\end{array}$ & $\stackrel{\curvearrowright}{m}$ & $\stackrel{\curvearrowright}{m}$ & 요 & $\underset{\infty}{\infty}$ & $\underset{\varpi}{\infty}$ & $\stackrel{\sim}{\sim}$ & 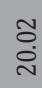 \\
\hline & 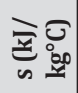 & & & $\begin{array}{l}\vec{A} \\
\stackrel{\sigma}{+}\end{array}$ & 命 & 文 & \begin{tabular}{l}
$\infty$ \\
2 \\
\multirow{2}{+}{} \\
6
\end{tabular} & 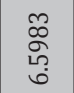 & क & 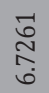 & 늠 & 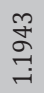 & $\begin{array}{l}\infty \\
\stackrel{0}{0} \\
\stackrel{0}{0}\end{array}$ & 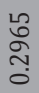 & 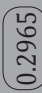 \\
\hline & $\underbrace{\sqrt[乛]{x}}_{a}$ & & & $\begin{array}{l}8 \\
\text { 음 }\end{array}$ & $\begin{array}{l}8 \\
\text { ํㅡㅁ }\end{array}$ & $\begin{array}{l}\text { 오 } \\
\text { m్- }\end{array}$ & ஓి & ஓి & 은 & 우 & $\begin{array}{l}\text { 농 } \\
\text { ஸे }\end{array}$ & 오 & 옹 & 눙 & $\begin{array}{l}\text { 오 } \\
\infty \\
0 \\
\text { 님 }\end{array}$ \\
\hline & $\underbrace{\sqrt{y}}_{=}$ & & & 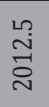 & ๙̊ & i̊ & $\underset{\sim}{\stackrel{+}{N}}$ & 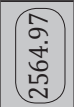 & 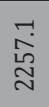 & $\begin{array}{l}\text { Бे } \\
\text { ñ } \\
\text { n }\end{array}$ & 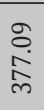 & 竞 & 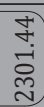 & $\begin{array}{l}\text { مू } \\
\text { ஸे }\end{array}$ & 守 \\
\hline & 을 & 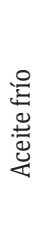 & 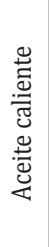 & 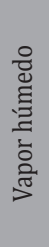 & 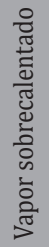 & 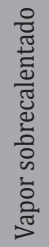 & $\frac{\check{\circ}}{\frac{\circ}{\pi}}$ & $\frac{\grave{\circ}}{\frac{\pi}{\pi}}$ & $\frac{\grave{\circ}}{\stackrel{\pi}{\pi}}$ & $\frac{\grave{\circ}}{\stackrel{\circ}{\pi}}$ & 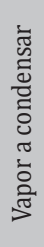 & 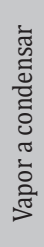 & 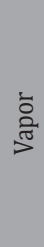 & 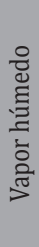 & ప్ర్య \\
\hline & 总 & $\dashv$ & $\sim$ & $m$ & 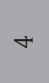 & in & $6^{-}$ & 0 & $N^{-}$ & $\Lambda$ & $\infty$ & $a$ & 욱 & $\exists$ & $\cong$ \\
\hline
\end{tabular}




\begin{tabular}{|c|c|c|c|c|c|c|}
\hline 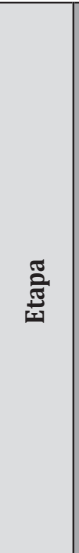 & 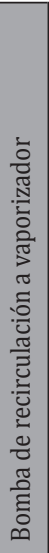 & 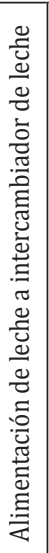 & 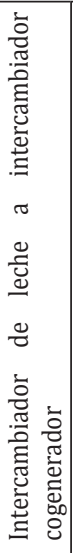 & 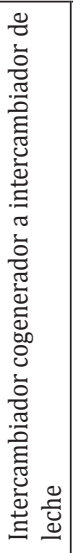 & 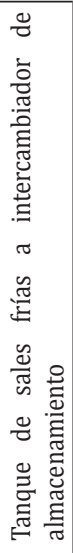 & 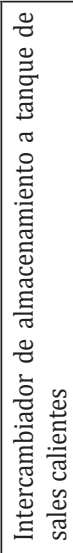 \\
\hline 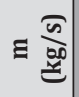 & $\begin{array}{l}\mathscr{L} \\
\dot{f}\end{array}$ & $\begin{array}{l}\stackrel{m}{*} \\
\underset{\forall}{\sim}\end{array}$ & $\begin{array}{l}\stackrel{m}{+} \\
\stackrel{+}{\sim}\end{array}$ & 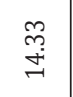 & $\begin{array}{l}\vec{\lambda} \\
\infty \\
\infty\end{array}$ & $\underset{\substack{\infty \\
\infty}}{\overline{1}}$ \\
\hline जे & & $\begin{array}{l}\infty \\
\dot{\infty} \\
\dot{m}\end{array}$ & $\begin{array}{l}\infty \\
\text { ஸे }\end{array}$ & $\begin{array}{l}\stackrel{\leftrightarrow}{\infty} \\
\dot{m}\end{array}$ & $\stackrel{\text { L }}{\longrightarrow}$ & $\stackrel{\text { L }}{\rightarrow}$ \\
\hline 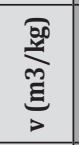 & $\begin{array}{l}\vec{m} \\
8 \\
0 \\
\overline{8} \\
0\end{array}$ & & & & $\begin{array}{l}\text { 농 } \\
\text { ᄋ } \\
\text { О }\end{array}$ & $\begin{array}{l}\text { Ln } \\
\stackrel{\circ}{0} \\
0\end{array}$ \\
\hline$\star$ & ' & & & & & \\
\hline$\underbrace{0}_{=}$ & $\begin{array}{l}\vec{\sigma} \\
\stackrel{\sqrt{v}}{v}\end{array}$ & $\sigma$ & $\stackrel{\infty}{m}$ & $\approx$ & 只 & 온 \\
\hline 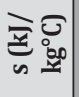 & \begin{tabular}{l}
$\frac{1}{0}$ \\
Oे \\
\multirow{0}{0}{}
\end{tabular} & & & & & \\
\hline 永 & 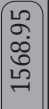 & & & & & \\
\hline 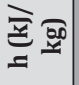 & 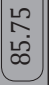 & & & & & \\
\hline 율 & 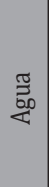 & 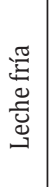 & 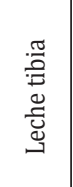 & 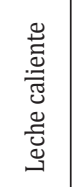 & 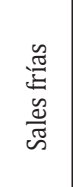 & 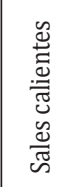 \\
\hline$\stackrel{\stackrel{\Xi}{\Xi}}{\Xi}$ & $\approx$ & $\stackrel{m}{\rightarrow}$ & $\underset{\sharp}{\sharp}$ & 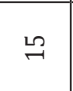 & $\underset{-1}{-1}$ & 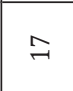 \\
\hline
\end{tabular}

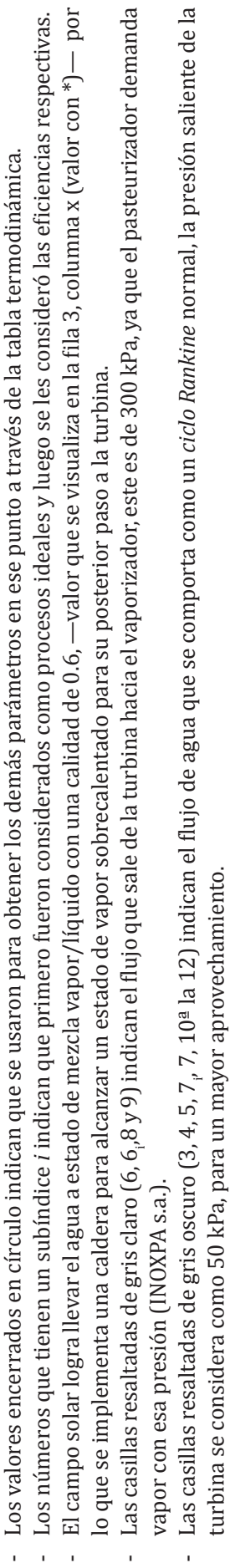




$$
\begin{gathered}
\text { Potencia }_{\text {vap }}=\left(h_{3}-h_{12}\right) \times \dot{m}_{3} \\
\text { Potencia }_{\text {vap }}=(2012.50 \mathrm{~kJ} / \mathrm{kg}-85.75 \mathrm{~kJ} / \mathrm{kg}) \times 46.60 \mathrm{~kg} / \mathrm{s} \\
\text { Potencia }_{\text {vap }}=89,793.10 \mathrm{~kW}
\end{gathered}
$$

Por lo que:

$$
\begin{gathered}
N_{\text {filas-panel }}=\frac{\text { Potencia }_{\text {vap }}}{N_{\text {paneles }} \times \text { Potencia }_{\text {panel }}} \\
N_{\text {filas-panel }}=\frac{89,793.10 \mathrm{~kW}}{4 \times 496.21 \mathrm{~kW}} \\
N_{\text {filas-panel }}=45.24 \text { filas }
\end{gathered}
$$

Se plantea utilizar 62 filas de paneles para así poder tener un sistema de almacenamiento térmico, pudiendo calcular la cantidad de paneles en total.

$$
\begin{gathered}
N^{\circ} \text { paneles }_{\text {total }}=62 \times 4 \\
N^{\circ} \text { paneles }_{\text {total }}=248 \text { paneles }
\end{gathered}
$$

Es importante mencionar que la cantidad de horas extra trabajadas estará de acuerdo a la irradiancia momentánea, por lo que la producción será variable en cada mes del año.

Teniendo en cuenta que el largo y ancho del panel es de 147.42 m y 5.76 $\mathrm{m}$ respectivamente, se puede estimar la superficie aproximada de nuestro campo solar.

Considerando que hay 4 paneles por cada fila y que estos se separan en un $10 \%$ de su longitud, el largo del campo solar sería:

$$
\begin{gathered}
\text { Largo }_{\text {camp.sol }}=147.42 \mathrm{~m} \times 4 \times 1.1 \\
\text { Largo }_{\text {camp.sol }}=648.65 \mathrm{~m}
\end{gathered}
$$

Asimismo, si tenemos 62 filas asumiremos que la separación entre estas es de la misma longitud del ancho del colector, por lo que la longitud ocupada por los colectores se multiplicaría por 2:

$$
\begin{gathered}
\text { Ancho }_{\text {camp.sol }}=5.76 \mathrm{~m} \times 62 \times 2 \\
\text { Ancho }_{\text {camp.sol }}=714.24 \mathrm{~m}
\end{gathered}
$$


Teniendo tanto el largo como el ancho del campo solar, podemos hallar su área:

$$
\begin{gathered}
\text { Area }_{\text {camp.sol }}=\text { Largo }_{\text {camp.sol }} \times \text { Ancho }_{\text {camp.sol }}=648.65 \mathrm{~m} \times 714.24 \mathrm{~m} \\
\text { Area }_{\text {camp.sol }}=463,290.35 \mathrm{~m}^{2}=46.33 \text { hectárea }
\end{gathered}
$$

Considerando un factor de servicio de $10 \%$, por los tanques y componentes que tiene nuestro campo solar, entonces:

$$
\begin{gathered}
\text { Area }_{\text {camp.sol }}=1.1 \times 46.33 \text { hectárea } \\
\text { Area Total } \text { camp.sol }_{\text {cón }}=50.96 \text { hectárea } \cong 51 \text { hectárea }
\end{gathered}
$$

Este valor será utilizado para el análisis económico.

En cuanto al almacenamiento térmico, este se dará con un tanque de sales de nitritos, ya que la temperatura a la que pueden llegar sin vaporizarse a una presión relativamente baja es de $500^{\circ} \mathrm{C}$; mucho mayor que en el caso del aceite térmico, considerando que el calor específico de las sales es de $1.5^{\circ} \mathrm{C}$, que la temperatura de entrada es de $250^{\circ} \mathrm{C}$, de salida de $500^{\circ} \mathrm{C}$, y que el mayor almacenamiento térmico que se obtendrá será en el mes de febrero, con un flujo de $94.01 \mathrm{~kg} / \mathrm{seg}$. Al considerar 11 horas de carga, el volumen necesario del tanque será de $2244 \mathrm{~m}^{3}$, pudiendo ser este un tanque de 9.83 metros de diámetro y 29.51 metros de alto.

A los 18 MW deberán restarse las potencias requeridas en las bombas de alimentación de aceite y de sales de nitratos.

$$
\begin{aligned}
& W_{\text {B aceite }}=v_{1} \times\left(P_{2}-P_{1}\right) \times \dot{m}_{1} / \eta_{\text {bomba }} \\
& W_{B \text { aceite }}=0.001212 \mathrm{~m}^{3} / \mathrm{kg} \times(2000 \mathrm{kPa}-100 \mathrm{kPa}) \times 290.25 \mathrm{~kg} / \mathrm{s} / \\
& W_{\text {B aceite }}=786.41 \mathrm{~kW} \\
& W_{\text {B nitrito }}=v_{16} \times\left(P_{17}-P_{16}\right) \times \dot{m}_{16} \\
& W_{\text {B nitrito }}=0.0005^{3} / \mathrm{kg} \times(1000 \mathrm{kPa}-100 \mathrm{kPa}) \times 88.71 \mathrm{~kg} / \mathrm{s} / \\
& W_{B \text { nitrito }}=51.47 \mathrm{~kW}
\end{aligned}
$$


Teniendo calculadas estas potencias, se determina también la energía necesaria por el enfriador o chiller para reducir la temperatura de los 50,000 $\mathrm{L} / \mathrm{h}$ leche de 38 a $4^{\circ} \mathrm{C}$, entonces:

$$
W_{\text {enf.leche }}=3.89^{\mathrm{kJ}} / \mathrm{kg} .{ }^{\circ} \mathrm{C} \times\left(38^{\circ} \mathrm{C}-4{ }^{\circ} \mathrm{C}\right) \times 50,000 \mathrm{~L} / \mathrm{h}
$$

Se transforma la unidad de flujo másico para que sea compatible con el resto de variables de la ecuación:

$$
\begin{gathered}
W_{\text {enf.leche }}=3.89^{\mathrm{kJ}} / \mathrm{kg} .{ }^{\circ} \mathrm{C} \times\left(38{ }^{\circ} \mathrm{C}-4{ }^{\circ} \mathrm{C}\right) \times 14.33 \mathrm{~kg} / \mathrm{s} \\
W_{\text {B leche }}=1897.54 \mathrm{~kW}
\end{gathered}
$$

Se transforma el resultado en unidades más comunes para selección de chillers.

$$
W_{\text {enf.leche }}=539.56 \text { Ton Ref. }=1^{\prime} 631,590.74 \mathrm{kcal} / \mathrm{h}
$$

Entonces, procedemos a seleccionar un grupo de chillers que puedan cumplir con la capacidad calculada, siendo este el HTS-150A, con una capacidad de 448,748 kcal /hr (Alibaba 2018); entonces:

$$
\begin{gathered}
N^{\circ} \text { Chillers }=1^{\prime} 631,590.74 \mathrm{kcal} / \mathrm{h} / 448,748 \mathrm{kcal} / \mathrm{h} \\
N^{\circ} \text { Chillers }=3.636 \cong 4 \text { chillers }
\end{gathered}
$$

Habiendo seleccionado los equipos, se considera su potencia máxima de trabajo, que es de $131.6 \mathrm{~kW}$. Multiplicamos este valor por 4 (cantidad de unidades):

$$
\begin{gathered}
W_{\text {total-chillers }}=131.6 \mathrm{~kW} \times 4 \\
W_{\text {total-chillers }}=526.4 \mathrm{~kW}
\end{gathered}
$$

Siendo una potencia útil global de:

$$
\begin{gathered}
W_{\text {util global }}=18000 \mathrm{~kW}-(786.4055 \mathrm{~kW}+51.47 \mathrm{~kW}+526.4 \mathrm{~kW}) \\
W_{\text {util global }}=16,635.73 \mathrm{~kW}
\end{gathered}
$$

Siendo esta potencia la considerada para hallar la energía generada total.

A continuación, se muestra una tabla de las horas extras trabajadas por el almacenamiento térmico, la energía generada y la leche pasteurizada en relación a cada mes del año: 
Tabla 3:

Energía generada y leche procesada por mes

\begin{tabular}{|l|c|c|c|}
\hline \multicolumn{1}{|c|}{ Mes } & $\begin{array}{c}\text { Horas de } \\
\text { almacenamiento por } \\
\text { día }\end{array}$ & $\begin{array}{c}\text { Energía Generada } \\
\text { (MwH) }\end{array}$ & $\begin{array}{c}\text { Leche Procesada } \\
\text { (Toneladas) }\end{array}$ \\
\hline Enero & 4.08 & 7,774 & 24,114 \\
\hline Febrero & 4.32 & 7,135 & 22,133 \\
\hline Marzo & 4.09 & 7,781 & 24,134 \\
\hline Abril & 3.07 & 7,021 & 21,776 \\
\hline Mayo & 1.71 & 6,556 & 20,336 \\
\hline Junio & 0.92 & 5,951 & 18,458 \\
\hline Julio & 1.30 & 6,342 & 19,670 \\
\hline Agosto & 2.54 & 6,983 & 21,659 \\
\hline Setiembre & 3.78 & 7,378 & 22,885 \\
\hline Octubre & 4.31 & 7,898 & 24,497 \\
\hline Noviembre & 4.17 & 7,571 & 23,484 \\
\hline Diciembre & 3.95 & 7,709 & 23,911 \\
\hline & TOTAL: & $\mathbf{8 6 , 0 9 9}$ & $\mathbf{2 6 7 , 0 5 7}$ \\
\hline
\end{tabular}

Fuente: Elaboración propia

\section{Análisis costo-beneficio}

Habiendo obtenido estos datos, se procede a realizar un análisis económico ${ }^{7}$ con el fin de sustentar la viabilidad del proyecto, por lo que se harán las siguientes consideraciones:

7 Siguiendo, en parte, los trabajos realizados por Caminero (2014) y González et al. (2014). 
Tabla 4:

Costos del proyecto (en US\$)

\begin{tabular}{|c|c|c|c|c|c|}
\hline Concepto & Descripción & Cantidad & Unidad & $\begin{array}{l}\text { Precio/ } \\
\text { Unidad }\end{array}$ & Total \\
\hline Terreno & $\begin{array}{l}\text { Terreno de captadores } \\
\text { solares }\end{array}$ & 51 & hectárea & 50,000 & $2,550,000$ \\
\hline \multirow{8}{*}{$\begin{array}{l}\text { Equipos de } \\
\text { generación } \\
\text { eléctrica }\end{array}$} & Turbina de vapor & 1 & unidades & $3,125,000$ & $3,125,000$ \\
\hline & Condensador & 1 & unidades & 150,000 & 150,000 \\
\hline & $\begin{array}{l}\text { Líneas de vapor y } \\
\text { condensado }\end{array}$ & 1 & unidades & 300,000 & 300,000 \\
\hline & Instrumento y Control & 1 & unidades & 37,500 & 37,500 \\
\hline & Caldera & 1 & unidades & 150,000 & 150,000 \\
\hline & Intercambiador de calor & 3 & unidades & 180,000 & 540,000 \\
\hline & Generador & 1 & unidades & 300,000 & 300,000 \\
\hline & Bombas & 1 & unidades & $1,200,000$ & $1,200,000$ \\
\hline \multirow{9}{*}{ Colector solar } & Reflectores & 83,328 & unidades & 48 & $3,999,744$ \\
\hline & Tubos receptores & 8,928 & unidades & 420 & $3,749,760$ \\
\hline & $\begin{array}{l}\text { Control de seguimiento } \\
\text { solar }\end{array}$ & 248 & unidades & 1,800 & 446,400 \\
\hline & $\begin{array}{l}\text { Accionamiento y } \\
\text { conexiones }\end{array}$ & 248 & unidades & 5,000 & $1,240,000$ \\
\hline & Estructura de soporte & 248 & unidades & 30,000 & $7,440,000$ \\
\hline & Cimentaciones & 248 & unidades & 13,500 & $3,348,000$ \\
\hline & Mano de Obra & 248 & unidades & 7,500 & $1,860,000$ \\
\hline & $\begin{array}{l}\text { Obras civiles, canaletas, } \\
\text { control general de campo } \\
\text { solar }\end{array}$ & 248 & unidades & 10,800 & $2,678,400$ \\
\hline & Aceite térmico & $15,951,773$ & $\mathrm{~kg}$ & 1 & $16,749,362$ \\
\hline Enfriamiento & Chiller 148.4 TR & 40,000 & unidades & 4 & 160,000 \\
\hline $\begin{array}{l}\text { Almacenamiento } \\
\text { térmico }\end{array}$ & Depósito de Sales & 365,933 & $\mathrm{kWh}$ & 30 & $10,978,003$ \\
\hline $\begin{array}{l}\text { Servicios } \\
\text { generales }\end{array}$ & $\begin{array}{l}\text { Ingeniería, supervisión, } \\
\text { aislamiento térmico }\end{array}$ & & & & $4,584,721$ \\
\hline \multicolumn{5}{|l|}{ TOTAL: } & $65,586,889$ \\
\hline
\end{tabular}

Fuente: Elaboración propia. 
Resulta así una inversión total de US\$65'586,889. Se plantea un 30\% de inversión inicial y un financiamiento por el porcentaje restante, para el cual consideramos una tasa de interés efectiva anual (TEA) de $5.5 \%$ y un tiempo de pago de 15 años. El tiempo de vida de una planta es de, por lo general, más de 30 años, por lo que consideraremos ese valor en nuestro flujo de caja.

Se está considerando además que el precio del gas natural es de US\$ $20.93 / 1000 \mathrm{~m}^{3}$, en función al rango de consumo de este combustible por mes. ${ }^{8}$ También se considera un alza del precio del gas natural en un $2.5 \%$ anual, y un crecimiento anual de 4\% en los gastos de operación y mantenimiento, ya que la planta se ira depreciando con el tiempo. A su vez, el precio de la energía eléctrica será de S/ $15.20,{ }^{9}$ y el precio por potencia será de S/ 20/ $\mathrm{kW}$-mes. ${ }^{10}$ La tasa de descuento para calcular el valor actual neto (VAN) se considerará en 5\% (Ver Anexo: Diagrama de flujo).

Habiendo aclarado la definición de valores en la tabla 5, los valores del VAN, con la tasa de descuento de $5 \%$, y de la tasa interna de retorno (TIR) son los siguientes:

- $\quad \mathrm{VAN}=\mathrm{US} \$ 2^{\prime} 499,660.54$

- $\quad \mathrm{TIR}=5.55 \%$

8 Conforme la Resolución OSINERGMIN 55-2018-OS/CD, que aprueba las Tarifas Únicas de Distribución de Gas Natural por Red de Ductos en Lima y Callao para el perÍodo 2018-2022.

9 Conforme al resumen de mercado libre de OSINERGMIN, correspondiente a junio de 2018. Véase: http://srvgart07.osinerg.gob.pe/SICLI/principal.aspx

10 Conforme la Resolución OSINERGMIN 56-2018-OS/CD, que fija precios en barra para el período mayo 2018-abril 2019. 


\section{Mecanismos de fomento estatal de los RER}

La promoción de la generación eléctrica con RER es básica para alcanzar un suministro seguro y ambientalmente eficiente. Ese es el objetivo principal del marco normativo de los RER en el Perú (Dammert et al. 2011). Además, los RER resultan adecuados «[...] para la consecución de los objetivos de la política energética [...] y [...] para la reducción de las emisiones de GEI», por lo que en Europa se promueve y fomenta su uso de forma normativa y jurisprudencial ${ }^{11}$ (Alenza et al. 2017: 459). En tal sentido, es necesario que el Estado fomente los RER para que sean sostenibles, en tanto que «concurre [en ellos] un interés público» (Sánchez 2017: 814). ${ }^{12}$

Al 2015, la energía termosolar representaba solo el $0.1 \%$ de la generación mundial de electricidad (IRENA 2018b). No obstante, según la International Renewable Energy Agency (IRENA), es necesaria una inversión para la descarbonización de la matriz energética, que considere el componente de energía solar concentrada (termosolar) (2018c).

Entre los instrumentos para fomentar los RER en el mundo, tenemos: (1) el sistema de tarifas, donde destaca el mecanismo de tarifas fijas (feed-intariff $)^{13}$ y el sistema de las primas; y (2) el sistema de cuotas, donde destacan las normas de cartera de renovables y el sistema de subastas. Además, los países establecen medidas para el fomento del mercado e incentivos fiscales (OSINERGMIN 2017). ${ }^{14}$

11 Entre la normativa, tenemos la Directiva 2009/28/CE del 23 de abril, relativa al fomento del uso de energía procedente de fuentes renovables, y la Directiva 2012/27/UE del Parlamento europeo y del Consejo del 25 de octubre, relativa a la eficiencia energética. Entre la jurisprudencia, tenemos la Sentencia del 13 de marzo de 2001, C-379/98 del Tribunal de Justicia de las Comunidades (Caso PreussenElektraAg) (Embid 2015: 147).

12 El agregado es nuestro.

13 Con ese fin, en Austria, se emitió el Ökostromgesetz, o «Ley de Energías Renovables de Austria»(Storr 2017).

14 Véase también: Haas et al. (2004) y véase: International Renewable Energy Agency (2018a). 
Gráfico 2:

Capacidad instalada de energía termosolar a nivel mundial

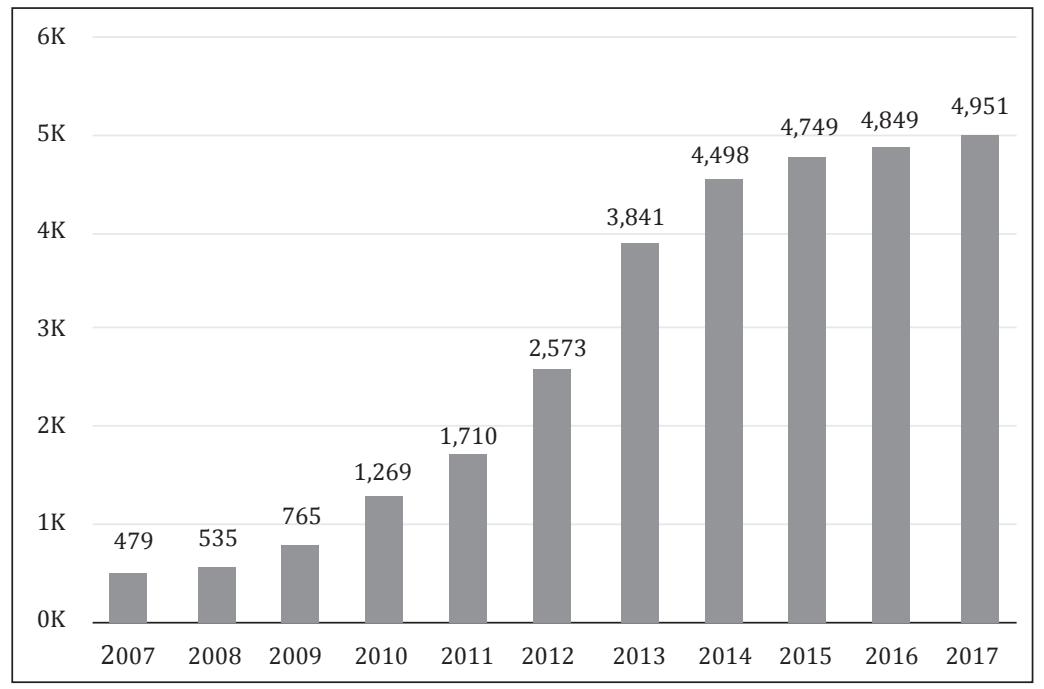

Fuente: IRENA: http://www.irena.org/solar. Fecha de consulta: 31 de agosto de 2018

En el Perú, la promoción de la generación con RER se da principalmente de tres maneras: (1) prioridad para el despacho diario de carga efectuado por el Comité de Operación Económica del Sistema Eléctrico Interconectado Nacional (COES), para lo cual se considera a la planta con un costo variable igual a cero; (2) una prima fijada por el OSINERGMIN que complementa el precio del Mercado de Corto Plazo, en caso que el costo marginal resulte menor que la tarifa determinada por el OSINERGMIN; (3) un mecanismo de subastas de primas gestionado por OSINERGMIN (artículos 5 y 7 del Decreto Legislativo 1002). ${ }^{15}$ Además, mediante la quinta disposición complementaria final de la «Ley Marco sobre Cambio Climático» se dispuso la creación de fondos de garantía para la promoción de inversión en RER. ${ }^{16}$

15 Véase también Dammert, A. (2009).

16 Sobre una experiencia de fondos públicos para impulsar las energías renovables, puede verse Zhao, Z. et al. (2011). 
Gráfico 3:

Potencia RER total subastada en Perú

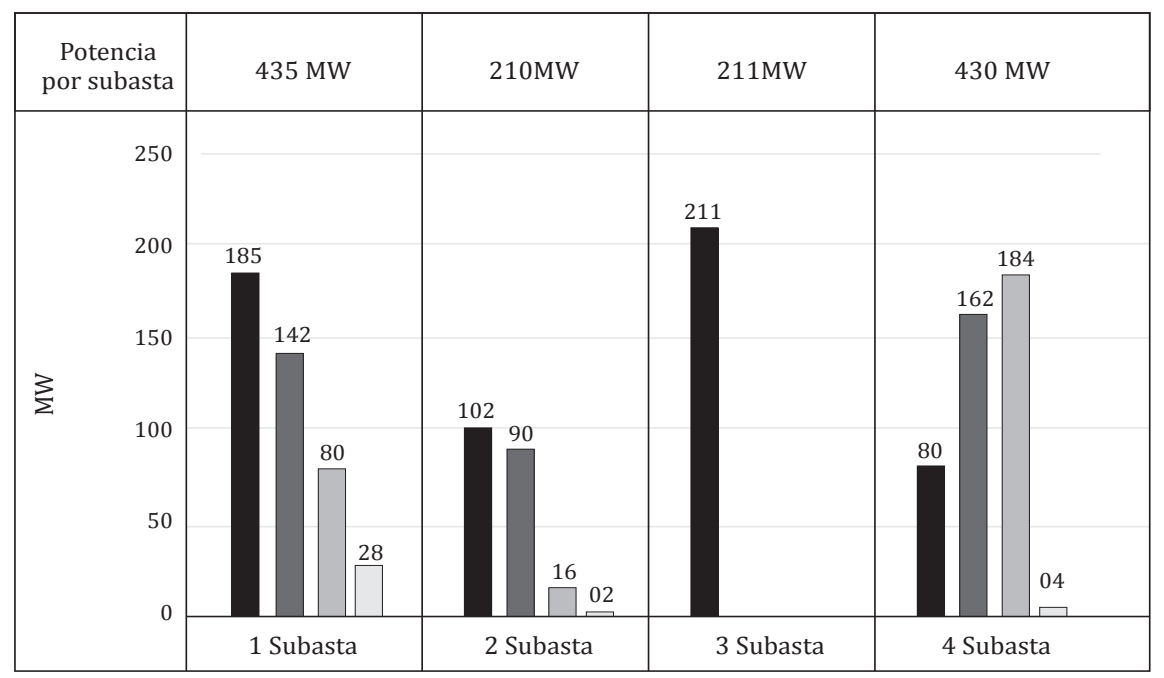

Hidro $\square$ Eólica $\square$ Solar $\square$ Biomasa

Elaborado por: Ministerio de Energía y Minas: https:/gestion.pe/economia/mem-alista-nuevo-esquema-promocion-energias-renovables-ano-227895. Fecha de consulta: 31 de agosto de 2018.

Por otro lado, la Ley 28832 fomenta la cogeneración, disponiendo que las centrales de este tipo podrán usar «[...] las redes de distribución pagando únicamente el costo incremental incurrido» (octava disposición complementaria final, inciso b). En caso estas centrales obtengan la condición de calificadas, contarán además con los beneficios que prevé el Reglamento de Cogeneración. ${ }^{17}$

Si bien la tecnología de cogeneración termosolar no se encuentra expresamente prevista en la normativa peruana, nuestra central es legalmente viable pues la fuente energética solar y la cogeneración sí se encuentran contempladas en la legislación, y son materia de fomento. Nuestra planta deberá obtener una autorización para que se pueda desarrollar la actividad de generación termoeléctrica. ${ }^{18}$ Incluso, nuestro proyecto podría

17 Principalmente un precio preferencial de gas natural (equivalente al cobrado a generadores eléctricos), prioridad en el despacho eléctrico y la aplicación de un peaje de conexión sin considerar su autoconsumo de potencia.

18 De acuerdo con el artículo 4 del Decreto Ley 25844, «Ley de Concesiones Eléctricas». «Se requiere autorización para desarrollar las actividades de generación termoeléctrica, cuando la potencia instalada sea superior a $500 \mathrm{KW}$. 
presentarse en una subasta RER, y se podría solicitar su consideración como central de cogeneración calificada ${ }^{19}$ para que obtenga los beneficios del Reglamento de Cogeneración.

\section{Conclusiones}

1. Los RER y la cogeneración son herramientas que los estados deben fomentar para hacer frente a los efectos del cambio climático, por ser tecnologías más limpias que las convencionales. En ese sentido, confluyen en ellas un interés público que justifica la actuación estatal.

2. El alto índice de radiación en Perú, más precisamente en Arequipa, nos permite unóptimoaprovechamiento de este tipo de energía, considerando también un correcto proceso de seguimiento solar continuo para poder llegar a altos valores de irradiancia aprovechada, siendo febrero el mes con mayor valor promedio, el cual es de $945.014 \mathrm{~W} / \mathrm{m}^{2}$.

3. Se obtuvieron los valores de las potencias que descuentan la potencia generada por nuestra planta, las cuales son las demandadas por la bomba de aceite en nuestro campo solar, la bomba de sales y el sistema de chiller requeridos para el enfriamiento de leche, cuyos valores son de $786.4055 \mathrm{~kW}, 51.47 \mathrm{~kW}, 526.4 \mathrm{~kW}$, respectivamente, por lo que se llega a una potencia útil total de $16,635.73 \mathrm{~kW}$

4. El proyecto es técnicamente viable, ya que nos permite generar una potencia eléctrica de $18 \mathrm{MW}$ en las horas de radiación y también en algunas horas extras por el almacenamiento térmico, sumando un total de 86,098.52 MWh anual. A su vez, el procesamiento de leche llegaría a un total de 267,056.79 toneladas por año, lo que conllevaría un gran ahorro de combustible fósil.

5. Se realizó un análisis económico teniendo en cuenta que el tiempo de vida de esta planta debería ser de 30 años e, incluso, superior. Considerando: (a) la cantidad de electricidad y potencia generadas, (b) el combustible ahorrado en el sistema del proceso de pasteurización y (c) el combustible gastado en la caldera de recalentamiento, se obtiene una alta rentabilidad con un VAN de US\$2'499,660.54 y un tiempo de recuperación de 19 años, con 11 años de ganancia pura para la industria.

6. Nuestra central de cogeneración solar es legalmente viable, pues tanto la fuente energética solar como la cogeneración se encuentran contempladas en la legislación y son promovidas por el Estado peruano. 


\begin{tabular}{|c|c|c|c|c|c|c|c|c|}
\hline 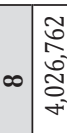 & 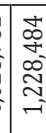 & $\begin{array}{l}\bar{Z} \\
b \\
0 \\
0\end{array}$ & 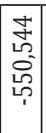 & 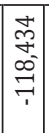 & 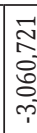 & 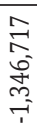 & 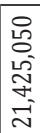 & 卓 \\
\hline & 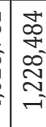 & $\begin{array}{l}m \\
\text { a } \\
\text { సे }\end{array}$ & 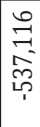 & 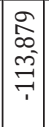 & \begin{tabular}{|l}
$\vec{N}$ \\
$\hat{D}$ \\
0 \\
$\tilde{m}$
\end{tabular} & 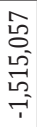 & 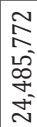 & 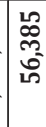 \\
\hline & 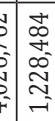 & \begin{tabular}{|cc}
$\widetilde{N}$ \\
\multirow{N}{N}{} \\
\end{tabular} & 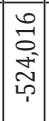 & $\mid \begin{array}{c} \\
\sigma \\
\sigma \\
o \\
o \\
7 \\
1\end{array}$ & 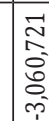 & $\begin{array}{l}\hat{\sigma} \\
\tilde{m} \\
\tilde{0} \\
0 \\
-1 \\
-1\end{array}$ & 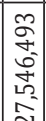 & : \\
\hline
\end{tabular}

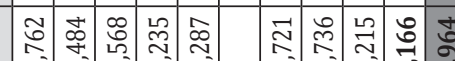

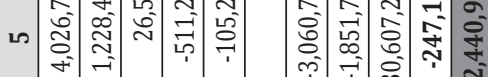

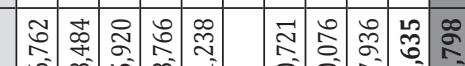

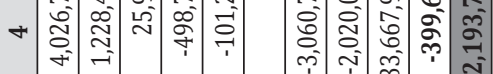

芯

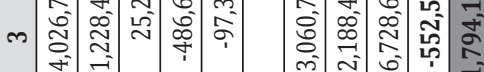

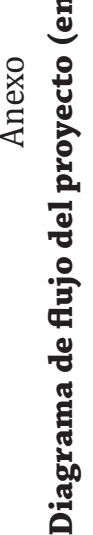

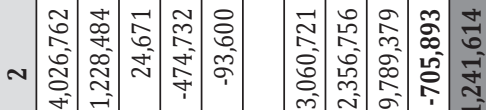
म-

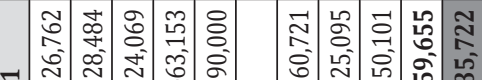

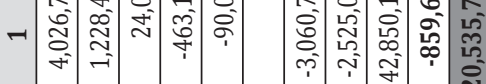

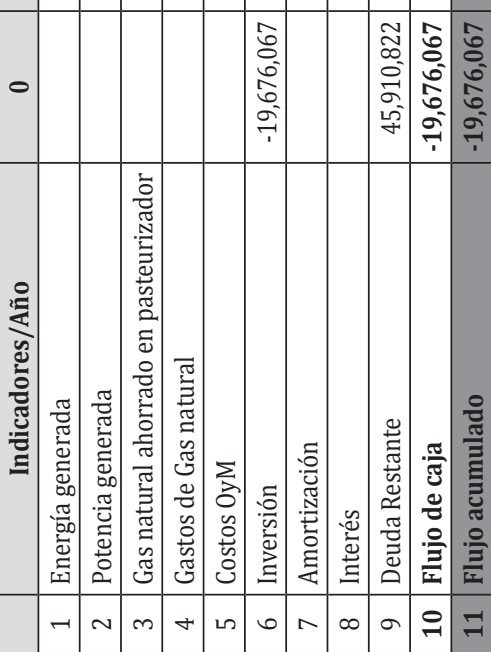

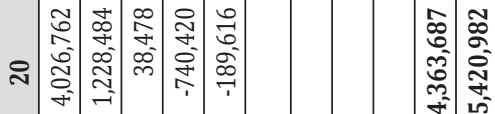

ปู

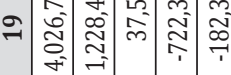

อิ จิ

萬

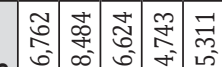

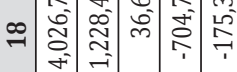

:-1:

Fे ले

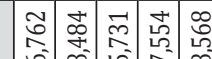

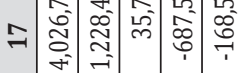

ฝี่ สู้

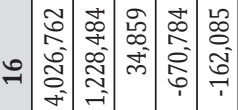

刅尔

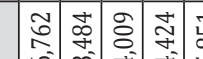

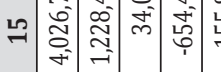

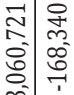

究

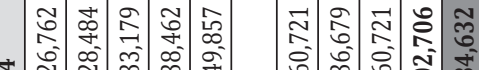

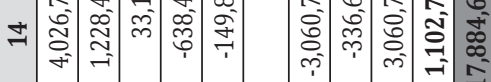

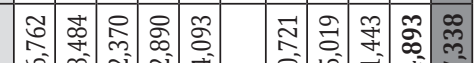

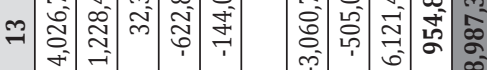

김

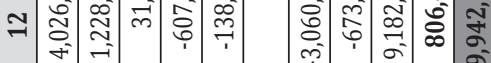

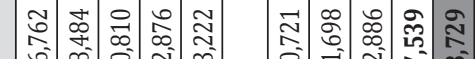

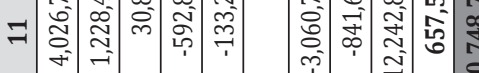

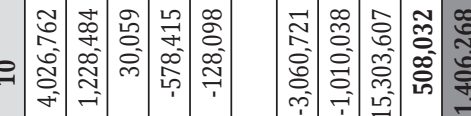

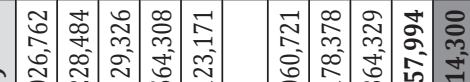

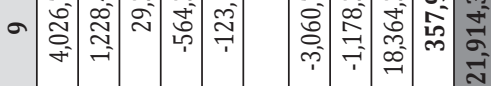

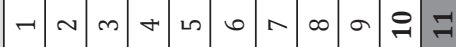




\begin{tabular}{|c|c|c|c|c|c|c|c|c|c|c|}
\hline ஓ्ल & 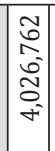 & 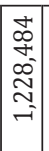 & 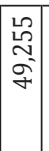 & 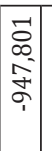 & 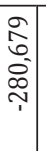 & & & & 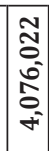 & 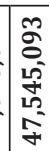 \\
\hline શิ & 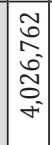 & 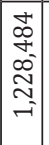 & 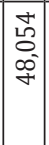 & 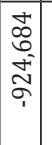 & 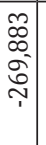 & & & & 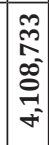 & 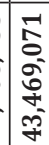 \\
\hline 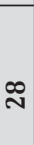 & 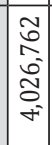 & 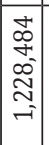 & 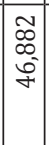 & 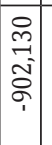 & 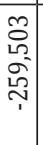 & & & & 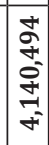 & 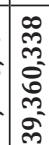 \\
\hline$\hat{N}$ & 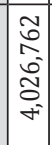 & 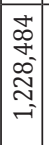 & 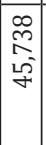 & 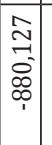 & 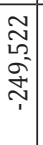 & & & & 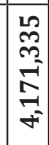 & 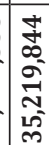 \\
\hline సి & 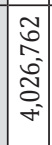 & 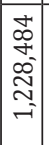 & 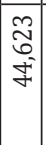 & \begin{tabular}{|l|}
$\vec{b}$ \\
0 \\
0 \\
0 \\
0 \\
0 \\
1
\end{tabular} & 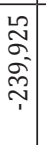 & & & & 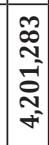 & 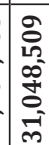 \\
\hline 논 & 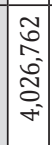 & 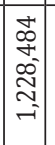 & 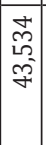 & \begin{tabular}{|c|}
$\infty$ \\
\\
$\stackrel{1}{\hat{N}}$ \\
$\infty$ \\
$\infty$ \\
1
\end{tabular} & 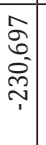 & & & & 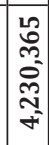 & 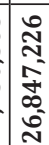 \\
\hline 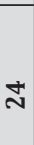 & 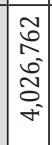 & 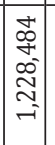 & \begin{tabular}{|c|}
\multirow{2}{*}{} \\
$\stackrel{+}{\checkmark}$ \\
\multirow{2}{*}{} \\
\end{tabular} & 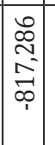 & 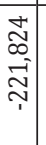 & & & & 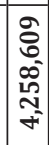 & $\begin{array}{c}-1 \\
0 \\
0 \\
0 \\
0 \\
0 \\
\text { ה్ }\end{array}$ \\
\hline$\tilde{N}$ & 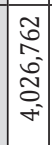 & 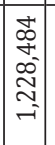 & 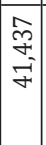 & 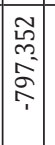 & 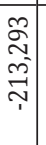 & & & & 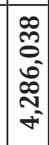 & 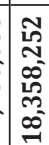 \\
\hline ลิ & 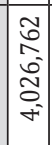 & 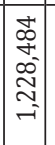 & 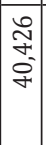 & 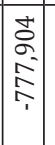 & 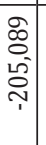 & & & & 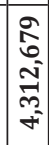 & 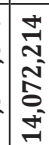 \\
\hline$\vec{N}$ & 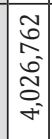 & 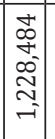 & 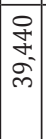 & 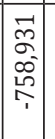 & 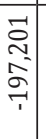 & & & & 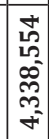 & 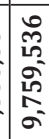 \\
\hline & 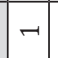 & $\sim$ & $m$ & + & in & o & $\wedge$ & 10 & 윽 & $\Rightarrow$ \\
\hline
\end{tabular}

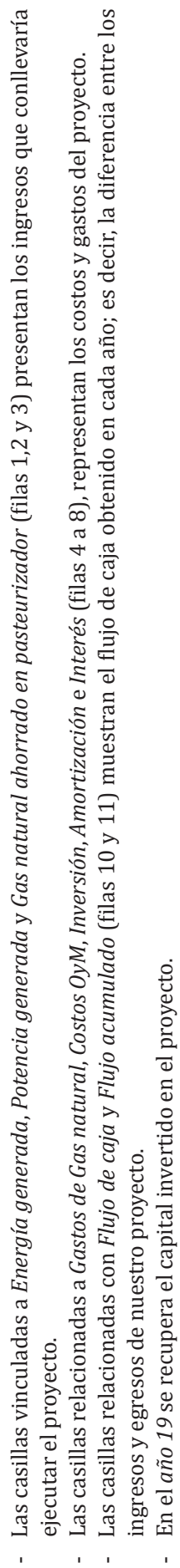




\section{Referencias}

ALEGRíA, D. (2016). «Caracterización numérica de un colector cilindro-parabólico de una central termosolar». Trabajo de fin de grado. Universidad de Cantabria. Recuperado de: https://repositorio.unican.es/xmlui/bitstream/handle/ 10902/8543/DAA.pdf?sequence=1

ALENZA, J. e I. SANZ (2017). «Las energías renovables y la eficiencia energética como instrumentos claves en la lucha contra el cambio climático». En Galán, R. e I. González (directores.). Derecho de las Energías Renovables y la Eficiencia Energética en el Horizonte 2020. Cizur Menor, Navarra: Thomson Reuters Aranzadi, pp. 451-484.

AlibabA (2018). «HTS-150A Air Cooled Screw Chiller». Recuperado de: https://www.alibaba. com/product-detail/Taiwan-brand-screw-compressor-150HP-air_60661002191. html?spm=a2700.7724838.2017115.42.1d702b2fsPY30t\&s=p

Bilgen, S., S. Keles, A. Kaygusuz, S. Ahmet y K. Kamil (2008). «Global warming and renewable energy sources for sustainable development: A case study in Turkey». Renewable and sustainable energy reviews, (12-2), pp. 372-396.

CAMinero, V. (2014). «Análisis económico de viabilidad de una planta termo-solar». Proyecto de Ingeniería. Universidad Pontificia Comillas. Recuperado de: https://repositorio.comillas.edu/xmlui/bitstream/handle/11531/1297/ PFC000046.pdf?sequence=1

Constitución Política del Perú. Separata del Diario Oficial El Peruano. Lima, 30 de diciembre de 1993.

DAMmERT, A. (2009). «Generación eléctrica con energías renovables no convencionales: el mecanismo de subastas». Revista de Derecho Administrativo (8), pp. 275-286.

Dammert, A., F. Molinelli, y M. Carbajal (2011). Fundamentos técnicos y económicos del sector eléctrico peruano. Lima: OSINERGMIN.

Dammert, A. y R. García (2017). Economía de la energía. Lima: Fondo editorial de la Pontificia Universidad Católica del Perú.

Domínguez, O, M. A. León, B. LóPez, B. MÉndeZ, F. MÉndez, M. G. Pinto, y L. ZÁRATE (2013). Propiedades Físicas de la leche. Recuperado de: https://es.slideshare. net/mercenaryy/propiedades-fisicas-de-la-leche-unidad-2

DeCREto LEgislativo 1002. Decreto Legislativo de promoción de la inversión para la generación de electricidad con el uso de energías renovables. Separata del Diario Oficial El Peruano. Lima, 2 de mayo de 2008.

Decreto Supremo 37-2006-EM. Aprueban sustitución del Reglamento de Cogeneración. Separata del Diario Oficial El Peruano. Lima, 7 de julio de 2006.

Decreto Supremo 12-2011-EM. Aprueban nuevo Reglamento de la Generación de Electricidad con Energías Renovables. Separata del Diario Oficial El Peruano. Lima, 23 de marzo de 2011.

DiARIo Gestión (2018). «MEM alista nuevo esquema de promoción de energías renovables para fin de año». Recuperado de: https://gestion.pe/economia/ mem-alista-nuevo-esquema-promocion-energias-renovables-ano-227895. Lima: 22 de febrero. 
Embid Irujo, A. (2015). «Energías renovables, medio ambiente y mercado interior de la energía: algunas reflexiones en las vísperas del cuarto paquete sobre la unión de la energía». En Embid Irujo, A. (coordinador). Agua, energía, cambio climático y otros estudios de derecho ambiental. En recuerdo a Ramón Mateo. Cizur Menor, Navarra: Thomson Reuters Aranzadi, pp. 133-160.

GoNZÁLEZ, I. (2011). Régimen jurídico-administrativo de las energías renovables y de la eficiencia energética. Cizur Menor, Navarra: Thomson Reuters Aranzadi.

GonzÁLEZ, J., J. Moreno, J. y S. SElva (2014). «Diseño y estudio económico de una planta termosolar de CCP para suministro de energía térmica a una planta láctea». Proyecto de Fin de Máster en Energías Renovables y Mercado Energético 2013/2014. Escuela de Organización Industrial. Recuperado de: https:// webcache.googleusercontent.com/search?q=cache:OlFu6zfOA2AJ:https:// www.eoi.es/es/file/20036/download\%3Ftoken\%3Dpz_1wYnc+\&cd=1\&hl= es\&ct=clnk\&gl=pe

HaAs, R., W. EichHammer C. Huber, O. LANGniss A. Lorenzoni y otros (2004). «How to promote renewable energy systems successfully and effectively». Energy Policy, (32), pp. 833-839.

INOXPA (s.a.). «Pasteurizador». Recuperado de: http://www.inoxpa.es/uploads/ document/Fitxes\%20t\%C3\%A8cniques/Equips/Pasteuritzador/ FTsolHTST.2_ES.pdf

International Renewable Energy Agency (2018). «Solar Energy Data». Recuperado de: https://www.irena.org/solar

International Renewable EnERgy Agency (2018a). Renewable energy auctions. Cases from sub-saharan Africa. Abu Dhabi, Emiratos Árabes Unidos.

InteRnational RENEWABle ENERgy Agency (2018b). Renewable energy policies in a time of transition. Abu Dhabi, Emiratos Árabes Unidos.

International Renewable Energy Agency (2018c). Global energy transformation. A roadmap to 2050. Abu Dhabi, Emiratos Árabes Unidos.

LEY 30754. Ley Marco sobre Cambio Climático. Separata del Diario Oficial El Peruano. Lima, 18 de abril de 2018.

LEY 28832. Ley para Asegurar el Desarrollo Eficiente de la Generación Eléctrica. Separata del Diario Oficial El Peruano. Lima, 23 de julio de 2006.

LóPEZ, E. (2000). «El protocolo de Kioto y su desarrollo en España. El fomento de las energías renovables y de la cogeneración eléctrica como instrumento de lucha frente al efecto invernadero». Documentación Administrativa (256), pp. 171-200.

Lüpfert, E., E. Zarza-Moya, M. Geyer, P. NAva, J. LANGenkamp y otros (2003). «Euro Trough Collector Qualification Complete - Performance Test Results from PSA». Conferencia del ISES Solar World Congress. Gotemburgo, Suecia, 1419 de junio. Recuperado de: https://elib.dlr.de/99768/1/EuroTrough_ Ises2003_0523_final_.pdf

MiNISTERIO DE INDUSTRIA, ENERGÍA Y TURISMO DE ESPAÑA (2016). «Evaluación completa del potencial de uso de la cogeneración de alta eficiencia y de los sistemas urbanos de calefacción y refrigeración eficientes». Madrid. Recuperado de: http://www.idae.es/file/13187/download?token=5T85pFgx 
Ministerio de Justicia y Derechos Humanos. «Sistema Peruano de Información Jurídica». Recuperado de: http://spij.minjus.gob.pe/normativa_libre/login.asp

MorA, M. (2014). «La ordenación jurídico-administrativa de las energías renovables como pieza clave en la lucha contra el cambio climático: ¿un sector en crisis?» Actualidad Jurídica Ambiental (17), pp. 1-22.

ObSERVATORIo de Energía RENovable PARA AmÉRICA LATINA y CARIBE (2013). «Energía Solar Térmica. Programa de Capacitación en Energías Renovables». Recuperado de: http://www.renenergyobservatory.org/applications/cbponre.html

OSINERGMIN (2016). La industria de la electricidad en el Perú. 25 años de aportes al crecimiento económico del país. Lima, Perú.

OSINERGMIN (2017). La industria de la energía renovable en el Perú. 10 años de contribuciones a la mitigación del cambio climático. Lima, Perú.

PAREDEs, C. (2012). «Diseño de captador solar cilíndrico parabólico para aplicaciones rurales en Paraguay». Proyecto final de carrera. Escuela Técnica uperior de Ingenieros de Minas, Asunción, Paraguay. Recuperado de: http://oa.upm. es/14011/1/PFC_CARMEN_PAREDES_VELASCO.pdf

PNUD (s.a.). Objetivos de Desarrollo Sostenible. Recuperado de: http://www.undp.org/ content/undp/es/home/sustainable-development-goals.html

RevistaEnergía.pe (2018). «Cerro Dominador, primer proyecto termosolar de Latinoamérica, inicia su última fase de construcción». Recuperado de: https://revistaenergia.pe/cerro-dominador-primer-proyecto-termosolarde-latinoamerica-inicia-su-ultima-fase-de-construccion/ Lima: 22 de agosto.

RESOLUCión OSINERGMIN 55-2018-OS/CD. Fijan la Tarifa Única de Distribución de Gas Natural en la Concesión de Lima y Callao aplicable al período comprendido entre el 07 de mayo de 2018 al 06 de mayo de 2022 y aprobación del Plan Quinquenal de Inversiones, el Plan de Promoción, y demás conceptos previstos en el Reglamento de Distribución. Separata del Diario Oficial El Peruano. Lima, 11 de abril de 2018.

RESOLUCIón OSINERGMIN 56-2018-OS/CD. Fija Tarifas en Barra para el período mayo 2018 abril 2019. Separata del Diario Oficial El Peruano. Lima, 13 de abril de 2018.

SÁnchez, M. (2017). Derecho Administrativo. Parte General. Decimotercera edición. Madrid: Tecnos.

SolANGi, K., M. R. IsLAm, R. SAidur, N. A. Rahim y H. FAYAZ (2011). «A review on global solar energy policy». Renewable and Sustainable Energy Reviews, vol. 15 (4), mayo, pp. 2149-2163.

STORR, S. (2017). «The status quo of renewable energy in Austria». En Galán, R. e I. González (directores). Derecho de las Energías Renovables y la Eficiencia Energética en el Horizonte 2020. Cizur Menor, Navarra: Thomson Reuters Aranzadi, pp. 117-128.

THERMinol (2015). «High performance under low-pressure applications. $-23^{\circ}$ to $325^{\circ}$ $C\left(-9^{\circ}\right.$ to $\left.620^{\circ} \mathrm{F}\right) »$. Recuperado de: http://emnmktassets.blob.core.windows. net/therminol/TF-03_Therminol_62_0.pdf

ZHAO, Z., J. Zuo, L.L. FAN y G. ZILLANTE (2011). «Impacts of renewable energy regulations on the structure of China - A critical analysis». Renewable Energy (36), pp. 24-30. 